\title{
Correction to: Numerical solution of space fractional diffusion equation by spline method combined with Richardson extrapolation
}

\section{Z. Soori ${ }^{1} \cdot$ A. Aminataei ${ }^{1}$}

Published online: 12 June 2020

○ SBMAC - Sociedade Brasileira de Matemática Aplicada e Computacional 2020

\section{Correction to: Computational and Applied Mathematics (2020) 39:136 https://doi.org/10.1007/s40314-020-01160-4}

In the Original publication the authors address were incorrectly published. The Correct address is given below.

1 "Faculty of Mathematics, K. N. Toosi University of Technology, P. O. Box: 1676-53381, Tehran, Iran"

Publisher's Note Springer Nature remains neutral with regard to jurisdictional claims in published maps and institutional affiliations.

The original article can be found online at https://doi.org/10.1007/s40314-020-01160-4.

\section{Z. Soori}

zsoori@mail.kntu.ac.ir
A. Aminataei

ataei@kntu.ac.ir

1 Faculty of Mathematics, K. N. Toosi University of Technology, P. O. Box: 1676-53381, Tehran, Iran 